\title{
Simulation of Nodules and Diffuse Infiltrates in Chest Radiographs Using CT Templates
}

\author{
G.J.S. Litjens ${ }^{1,2}$, L. Hogeweg ${ }^{2}$, A.M.R. Schilham², P.A. de Jong ${ }^{2}$, \\ M.A. Viergever ${ }^{2}$, and B. van Ginneken ${ }^{2,1}$
}

1 Diagnostic Image Analysis Group, Radboud University Nijmegen Medical Centre, The Netherlands

2 Image Sciences Institute, Utrecht University Medical Center, The Netherlands

\begin{abstract}
A method is proposed to simulate nodules and diffuse infiltrates in chest radiographs. This allows creation of large annotated databases for training of both radiologists and computer aided diagnosis systems. Realistic nodules and diffuse infiltrates were generated from three-dimensional templates segmented from CT data. These templates are rescaled, rotated, projected and superimposed on a radiograph. This method was compared, in an observer study, to a previously published method that simulates pulmonary nodules as perfectly spherical objects. Results show that it is hard for human observers to distinguish real and simulated nodules when using templates (AUC-values do not significantly differ from .5, $p>.05$ for all observers). The method that produced spherical nodules performed slightly worse (AUC of one observer differs significantly from $.5, p=.011)$. Simulation of diffuse infiltrates is challenging but also feasible ( $\mathrm{AUC}=0.67$ for one observer).
\end{abstract}

Keywords: CT, radiograph, simulation, nodules, diffuse infiltrates.

\section{Introduction}

Lung diseases are among the largest causes of death and disability in the world. In most cases early detection, for example in screening using computed tomography $(\mathrm{CT})$ or radiography, increases the chance of recovery significantly. Due to for example dose and cost considerations, chest radiography is by far the most common radiological exam [1]. It has been shown previously that in chest radiography detection of pulmonary nodules and the detection of focal shadowing is extremely difficult, even for expert radiologists [2]. For nodules it was found that when a nodule was missed, it was visible in retrospect in $90 \%$ of cases $[3$.

This shows how important it is to train radiologists in reading chest radiographs (CRs). Also, the development of computer aided diagnosis (CAD) systems to act as a first or second reader has been shown to improve accuracy [4]. For both radiologist and $\mathrm{CAD}$ training extensive databases with proven radiological findings are required, but constructing such databases is a laborious process.

In this paper a method is proposed to simulate lesions on CRs. This method uses CT templates of real nodules and diffuse infiltrates in combination with 


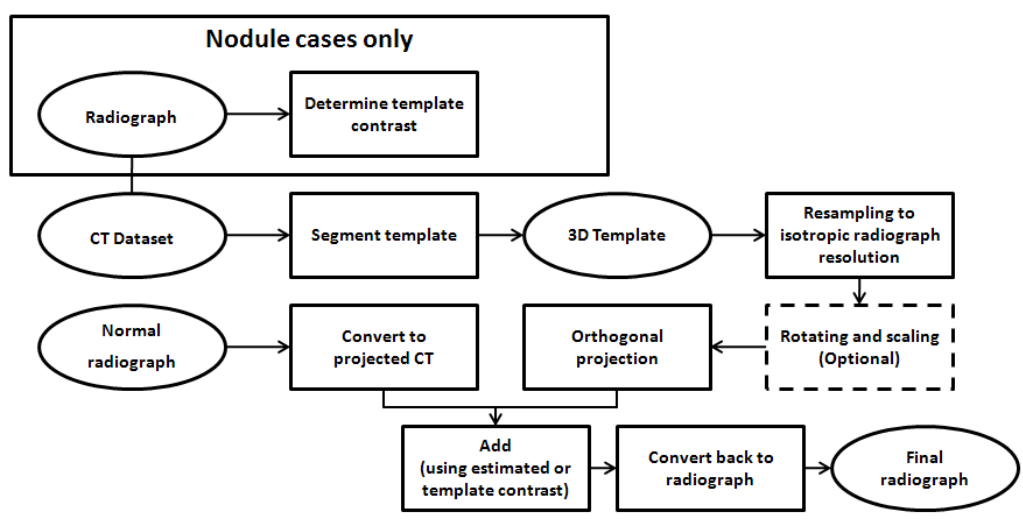

Fig. 1. Flowchart of the method for simulating abnormalities in radiographs

normal CRs which enables generation of large amounts of training data. Section 2 describes the data and Section 3 the method. In Section 4 the results of an observer study are presented that investigates if simulated lesions are indistinguishable from real pathology. Section 5 discusses the results and suggests topics for further research.

\section{Materials}

For the nodule templates radiographs and CT images from the NELSON lung cancer screening trial [5] were used. We used 36 pairs of radiographs and CT scans obtained within a three month period. The nodules in the radiographs were subsequently annotated by a radiologist using the CT scan as ground truth.

The templates for generating diffuse infiltrates were obtained from 44 clinical CT scans with corresponding radiographs. These radiographs were also annotated according to the findings in the CT scan. The annotation was done by an expert chest radiologist. 6

Finally, 42 normal radiographs images were selected from the lung cancer screening database. All radiographs in this study were acquired using a Philips Digital Diagnost with a cesium iodine scintillator, a $3000 \times 3000$ pixels acquisition matrix and $.143 \mathrm{~mm}$ resolution.

\section{Methods}

The proposed method is an extension and adaption of a previously published method by Schilham et al. [7. The simulation method can be summarized (Figure 1) as follows: a radiograph and a lesion template (nodule or diffuse infiltrate segmented from a CT scan) are needed. The segmented lesion is resampled with $3 \mathrm{D}$ cubic interpolation to an isotropic resolution equal to the pixel resolution of 


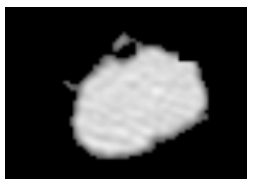

(a)

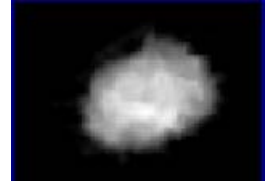

(b)

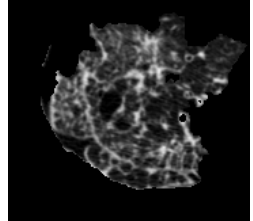

(c)

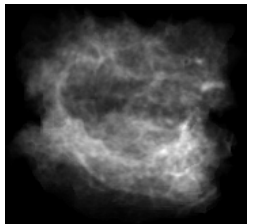

(d)

Fig. 2. Simulation templates: (a) CT slice of a segmented nodule, (b) Projection of a CT nodule, (c) CT slice of a segmented diffuse infiltrate and (d) Projection of a CT diffuse infiltrate

the radiograph. As an additional step the lesion can be rotated and scaled. Subsequently the pixel values of the radiograph are converted to projected Hounsfield units. The lesion is then projected to a $2 \mathrm{D}$ image and superimposed on a random location in this image using a contrast value. The final step is conversion from the projected CT values to pixel values of the radiograph.

CT template acquisition. The segmentation of the nodules from the CT was performed using a segmentation technique published by Kostis et al. 8. In some cases a segmentation was deemed unusable because of the shape of the nodule, e.g. in the case of a pleural lesion. This resulted in a final number of 20 templates.

Diffuse infiltrates were segmented using a previously developed semi-automatic method. Twenty-six diffuse infiltrate templates were obtained. Examples of templates are shown in Figure 2.

Preprocessing and projection of the template. The obtained templates were resampled to an (almost) isotropic resolution equal to the resolution of the radiograph pixels using cubic 3D interpolation. Next the template can be rotated and/or rescaled to allow the creation of a wide range of different lesions from a single template.

The template has to be projected to a 2D image to allow superimposition of the template on a radiograph. Using simple raycasting the lesion template can be projected to a $2 \mathrm{D}$ image.

Conversion from radiograph to projected CT. Projection of a CT data set should in theory be similar to a radiograph. In practice there are several reasons why this is not the case, such as different x-ray energies and proprietary post-processing algorithms applied by manufacturers to radiographs. To overcome these problems, a conversion step from the pixel values encountered in the radiograph to projected $\mathrm{CT}$ is needed.

In this study a simple intensity mapping is used:

$$
I_{\mathrm{CT}}=F\left[I_{r}\right],
$$

where $I_{r}$ and $I_{\mathrm{CT}}$ represent the intensity of a pixel in the radiograph and the projected CT, respectively, and $F$ is the mapping function. This mapping 


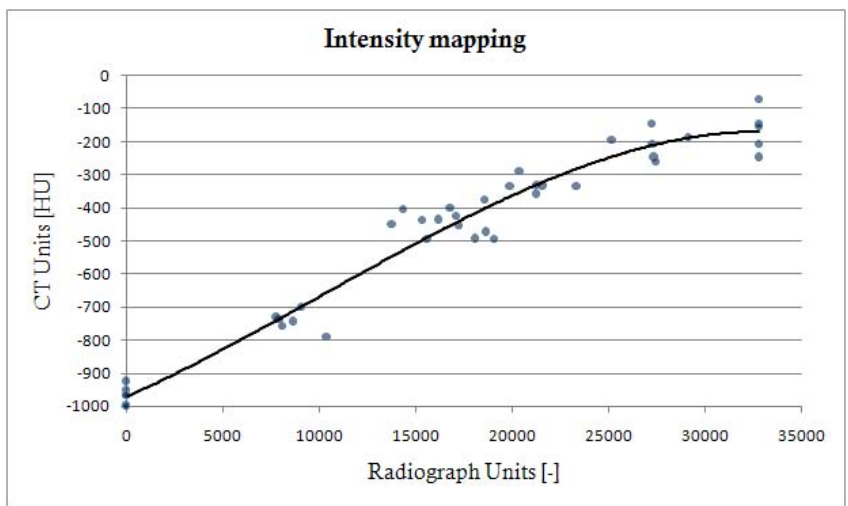

Fig. 3. Relation between intensities in radiographs and average projected CTs determined by a third-order polynomial. Data points were obtained from measurements in five radiographs and projected $\mathrm{CT}$ images.

function can be determined by measuring the intensities in both images in similar regions. The intensities were measured in the heart, in the spine and in the lung fields, and, additionally, the lowest and highest pixel value were added. These five values, determined in five radiographs and five corresponding projected CTs from the lung screening trial database, were used to fit a third-order polynomial, and this resulted in the mapping function, shown in Figure 3. It will not completely undo the effects of the more advanced post-processing steps (edge detection, unsharp masking) but it was adequate for the purpose of this study.

Superimposition. The final step is to add the projected template to the converted radiograph. Lung masks generated using a published method [9] were used to make sure that generated nodules were contained completely within the lung fields.

For diffuse infiltrate templates a different approach was used. A random position was selected within the lung mask. All the template pixels outside the mask were discarded. To remove edge artifacts, pixels close to the lung border (10 pixels, Euclidean distance) were added with reduced intensity (using an exponential falloff). The superimposition itself is straightforward. First a contrast measure is defined:

$$
c=\log \left[\frac{I_{t}}{I_{b}}\right],
$$

where $c$ is the template contrast and $I_{t}$ and $I_{b}$ are the mean template and background intensities, respectively. Next, for each template the mean background intensity $I_{b}$ and mean template intensity $I_{t}$ were measured in the radiograph belonging to the CT scan the template was segmented from. This resulted in a contrast value $c$ for each template. When superimposing the template on a radiograph, this $c$ is used in conjunction with the mean background intensity at 
the selected template position to determine what the mean template intensity $I_{t}$ should be to achieve the same contrast. The template mean is then adjusted to this value. This allows realistic superimposition of templates.

For nodules it is easy to implement this automatically because the standard deviation on $I_{b}$ is small due to the relatively small size of the templates. However, for diffuse infiltrate templates this is more difficult as they can span a large region of the lung, and the contrast can vary considerably within that region. Therefore the contrast values of simulated diffuse lesions were adjusted manually in this study. Some simulation results are shown in Figure 4.

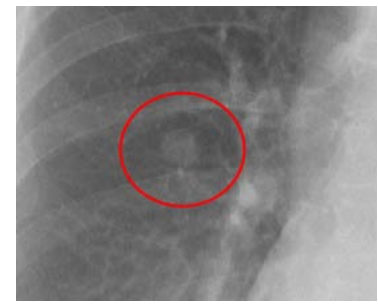

(a)

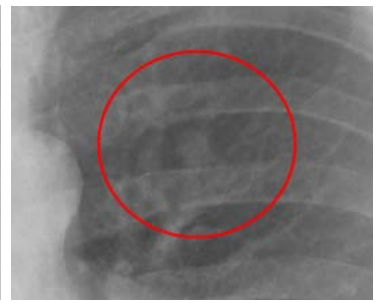

(b)

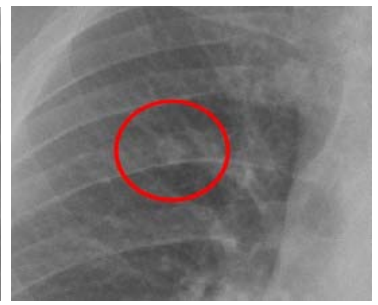

(c)

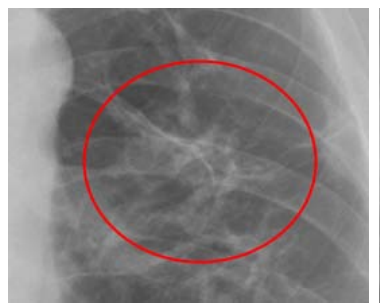

(d)

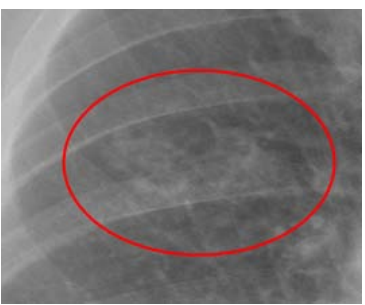

(e)

Fig. 4. Real lesions and simulation examples: (a) Real nodule, (b) Template based lesion simulation, (c) Perfectly spherical object nodule simulation, (d) Real diffuse infiltrate and (e) Simulated diffuse infiltrate

Perfect spherical nodule simulation. Samei et al. [10 have validated a different method for simulation of nodules in CRs. This method uses a mathematical representation of a nodule contrast profile. This profile is based on measurements in a real nodule database and the formula for this mask is:

$$
c[r]=C\left(\frac{4}{D^{4}} r^{4}-\frac{4.2}{D^{2}} r^{2}+1\right)
$$

Here $r$ is the position along the radius, $C$ is the peak contrast value and $D$ is the diameter. The definition of contrast is the same as in Eq. 2. Using a value for $c$ and $D$, a simulated nodule can be added to a radiograph (Fig. 4c). 
Table 1. Area under the curve and corresponding p-values (AUC / p-value) when compared to $\mathrm{AUC}=.5$ for all observers

\begin{tabular}{c|c|c|c} 
Observer & All nodules & Template method & Samei's method \\
\hline 1 & $.59 / .2$ & $.49 / 1$ & $.66 / .01$ \\
\hline 2 & $.40 / .1$ & $.39 / .2$ & $.41 / .2$ \\
\hline 3 & $.57 / .3$ & $.56 / .5$ & $.59 / .2$
\end{tabular}

\section{Experiments and Results}

To investigate if the template based lesion simulation can generate realistic nodules and diffuse infiltrates in radiographs, two observer studies were performed. Three observers participated in the studies. Observer 1 and 2 were image analysis scientists with a expertise in chest $\mathrm{CT}$ and radiography. Observer 3 was a radiologist specializing in chest radiology. Whether a simulated lesion was realistic was determined using an analog scale from 1 to 100, represented as a slider to the observers.

In the first experiment 39 real nodules were used. Using template based lesion simulation a set of 39 nodules was simulated. In addition, using the perfectly spherical nodule simulation method, another 39 nodules were simulated. The parameters used for perfectly spherical nodule generation were a nodule diameter $D$ between 7 and $22 \mathrm{~mm}$ and a peak contrast value $C$ of between .15 and .22 , which was comparable to our templates. This resulted in a total of 117 nodules, of which 78 are simulated, 39 for each method. In the second experiment the hypothesis that simulated and real diffuse infiltrates were indistinguishable is tested. To this end, 23 images containing diffuse infiltrates were acquired from clinical practice for which annotations by an expert radiologist were available. In these images between two and four regions were selected for scoring, leading to a total of 66 regions. In addition, diffuse infiltrate templates were superimposed on 23 normal radiographs, varying between 3 and 8 templates per image to get a good coverage of the lung. In these radiographs, 2 to 4 regions were annotated, leading to a total of 63 regions. The results are presented as receiver operating characteristic (ROC) curves in figure 5. A non-parametric method [1] is used to determine if the area under the curve (AUC) differs significantly from .5. Table 1 shows the AUC and p-values of all curves on nodule simulation.

Observer 1 was the only observer to achieve an AUC significantly larger than chance performance in the nodule experiments, when comparing real nodules with the nodules simulated as perfect spheres. This observer performed the study on diffuse infiltrates and attained an AUC of .67, significantly higher than .5 $(p=.001)$, this is shown in figure $5 \mathrm{~d}$.

\section{Discussion and Conclusion}

The results show that it is difficult for the observers to distinguish real and simulated nodules. Although both observer 1 and 3 do have a AUC higher than .5 
for the all-nodule case, the p-values indicate that this difference is not significant $(p>.05)$. Figure $5 \mathrm{~b}$ and $\mathrm{c}$ show the results for the methods separately. It can be seen that observer 1 and 3 perform better on nodules simulated with the perfectly spherical object nodule method. These nodules seem less realistic than those obtained by the template based lesion simulation method presented here. This can also be seen in table 1, where for the spherical nodules the AUC is significantly different from .5 $(p<.05)$ for observer 1 . No significant difference is found between the AUC and an AUC of .5 for nodules simulated with the template method for all observers $(p>.05)$. Observer 1 was asked to participate in the diffuse infiltrate experiment as he performed the best on the nodule cases. His results show a significant difference between his AUC of .67 and an AUC of $.5(p=.001)$. This means that observer 1 can see the difference between some

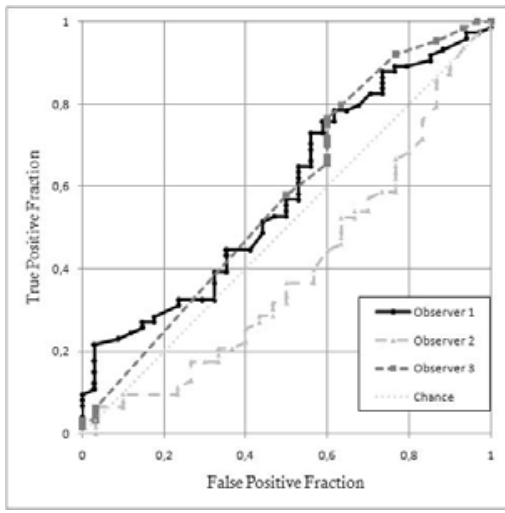

(a)

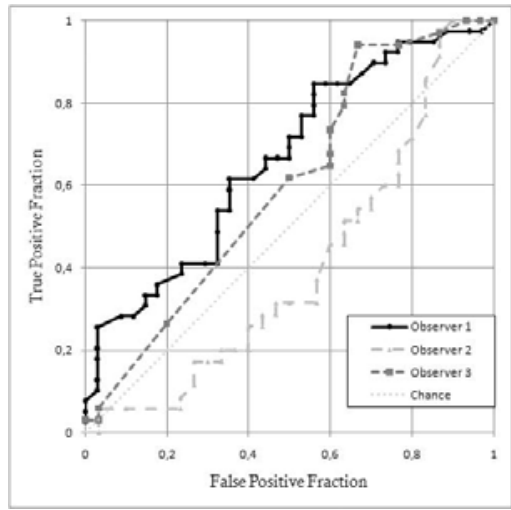

(c)

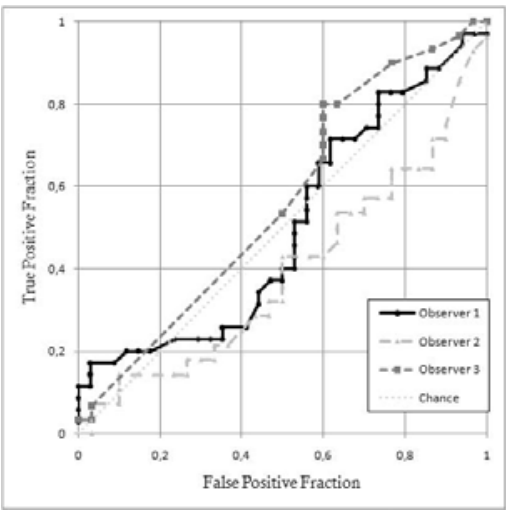

(b)

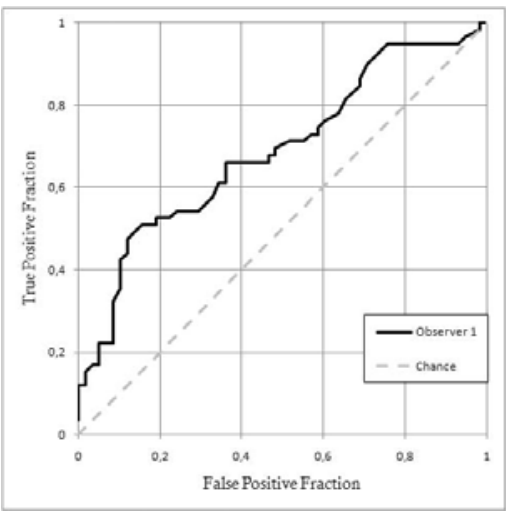

(d)

Fig. 5. ROC curves of the observer studies performed on distinguishing simulated lesions from real lesions: (a) Observer ROC curve of all nodules, (b) ROC curve of nodules simulated with the template method, (c) ROC curve of nodules simulated with perfectly spherical object method and (d) ROC curves for diffuse infiltrates 
real and simulated diffuse infiltrates. As the AUC is still low, it seems plausible that diffuse infiltrates can be generated.

Future work should focus on generating a contrast function to allow better superimposition of especially diffuse infiltrates. Creating a realistic contrast between lesion and background is the most challenging aspect in these simulations. Also, the effect of post-processing could be investigated by using raw radiographs. It could also be helpful to collect more templates for diffuse infiltrates which exhibit a large variation in appearance.

Concluding, it is possible to simulate nodules in radiographs that are indistinguishable from real nodules. Using a small set of templates, a wide range of lesions can be created. For diffuse infiltrates, results show that many simulated lesions are realistic, which is an encouraging result. The proposed method is the first method that allows the simulation of diffuse infiltrates in CRs, and for simulating nodules it is more effective than previously published methods.

\section{References}

1. Bhargavan, M.: Trends in the utilization of medical procedures that use ionizing radiation. Medical Physics 134, 612-627 (2008)

2. Forrest, J.V., Friedman, P.J.: Radiologic errors in patients with lung cancer. Western Journal of Medicine 134, 485-490 (1981)

3. Muhm, J.R., Miller, W.E., Fontana, R.S., Sanderson, D.R., Uhlenhopp, M.A.: Lung cancer detected during a screening program using four-month chest radiographs. Radiology 148, 609-615 (1983)

4. Abe, H., Macmahon, H., Engelmann, R., et al.: Computer-aided Diagnosis in Chest Radiography: Results of Large-Scale Observer Tests at the 1996-2001 RSNA Scientific Assemblies. Radiographics 23, 255-265 (2003)

5. Xu, D.M., Gietema, H., de Koning, H., Vernhout, R., Nackaerts, K., Prokop, M., Weenink, C., Lammers, J., Groen, H., Oudkerk, M., van Klaveren, R.: Nodule management protocol of the NELSON randomised lung cancer screening trial. Lung Cancer 54(2), 177-184 (2006)

6. Arzhaeva, Y., Prokop, M., Tax, D.M.J., de Jong, P.A., Schaefer-Prokop, C.M., van Ginneken, B.: Computer-aided detection of interstitial abnormalities in chest radiographs using a reference standard based on computed tomography. Medical Physics 34(12), 4798-4809 (2007)

7. Schilham, A.M.R., van Ginneken, B.: Simulating nodules in chest radiographs with real nodules from multi-slice CT images. In: Medical Imaging (2006)

8. Kostis, W., Reeves, A., Yankelevitz, D., Henschke, C.: Three-dimensional segmentation and growth rate estimation of small pulmonary nodules in helical CT images. Trans. Med. Imag. 22(10), 1259-1274 (2003)

9. van Ginneken, B., Stegmann, M.B., Loog, M.: Segmentation of anatomical structures in chest radiographs using supervised methods: a comparative study on a public database. Medical Image Analysis 10(1), 19-40 (2006)

10. Samei, E., Flynn, M.J., Eyler, W.R.: Detection of Subtle Lung Nodules: Relative Influence of Quantum and Anatomic Noise on Chest Radiographs. Radiology 213(3), 727-734 (1999)

11. DeLong, E., DeLong, D., Clarke-Pearson, D.: Comparison of the area under two or more correlated receiver operating characteristic curves. Biometrics 44, 837-845 (1988) 